\title{
A link between short-range and long-range properties of random sphere packings
}

\author{
R. Balzan - A. L. Sellerio - D. Mari • \\ A. Comment - G. Gremaud
}

Received: 21 May 2013 / Published online: 6 October 2013

(C) Springer-Verlag Berlin Heidelberg 2013

\begin{abstract}
We present a high precision particle-by-particle $3 \mathrm{D}$ reconstruction of granular systems composed of monodispersed spheres (sphere packings); the experimental approach is based on magnetic resonance imaging techniques. Our measurements revealed a strong correlation between the volume defined by the distance to the first nearest neighbor and the long-range average density. The main contribution to the amplitude decay of the correlation function can be described as exponential rather than power law up to a range equal to 7 sphere diameters. No evidence of geometrical structural changes as a function of the density was observed and neither regular crystallites nor any other statistically significant structures could be ascribed to a specific local arrangement. We concluded that granular compaction is the result of a process through which the system changes the average size of local structures without changing their local geometrical characteristics. These conclusions are supported by two-body correlation functions and Voronoi polyhedra space decomposition. The results
\end{abstract}

R. Balzan and A. L. Sellerio equally contributing authors.

Electronic supplementary material The online version of this article (doi:10.1007/s10035-013-0450-8) contains supplementary material, which is available to authorized users.

R. Balzan $(\bowtie) \cdot$ A. Comment

Institute of Physics of Biological Systems,

EPFL, 1015 Lausanne, Switzerland

e-mail: riccardo.balzan@epfl.ch

A. L. Sellerio · D. Mari · G. Gremaud

Laboratoire de Physique de la Matière Complexe,

EPFL, 1015 Lausanne, Switzerland

\section{A. L. Sellerio}

IENI-CNR, 20125 Milano, Italy provide a different perspective on the mechanisms underlying compaction with respect to previous works, and allow to discriminate between the different existing theoretical approaches.

Keywords Compaction - Magnetic resonance imaging (MRI) - 3D image reconstruction · Bulk structure .

Voronoi decomposition · Correlation function

Granular materials are complex systems composed of a large number of particles interacting mainly via contact forces [13]. Experimentally, granular systems show a rich and complex behavior (e.g. self-organized criticality [4], nonlinear dynamics [5,6] and jamming [7]). Granular media can also be used to model even more complicated problems, such as glasses [8]. The first studies on the problem of sphere packings date back to Kepler's work [9]. In modern times, the seminal papers by Bernal [10] and Scott [11] introduced the main mathematical tools and concepts now common to the field. In recent years this field has seen a renewed interest $[12,13]$ : several studies tackled open problems such as the nature of sphere-sphere correlation, the geometric properties of the first shell (defined by the distance and arrangement between nearest neighbors), and the onset of crystallization with respect to compaction; the studies spanned from experimental investigations using MRI $[6,14]$ or other techniques [15-17] to theoretical approaches [18-22] and simulations $[23,24]$.

These systems have been widely studied experimentally from a rheological and structural as well as from a theoretical point of view, focusing on the large-scale properties of the samples, the short-scale geometric arrangements and the nature of the physical interactions between the particles that are nonetheless crucial since they define the local properties, which in turn define the global static and dynamic 
behavior. Granular systems are also a fertile field for numerical approaches.

To focus on the structural aspect, we obtained full tomographic views, by means of MRI [25], of packings composed of around $2 \cdot 10^{4}$ monodispersed $3 \mathrm{~mm}$ diameter $D$ polymer spheres (Fig. 1a). The output 3D matrix consisted of a water density map with an isotropic resolution of $0.2 \mathrm{~mm} \approx D / 15$. The spheres were immersed in a cylindrical container filled with water doped with paramagnetic relaxing agents (copper aqua ions; see Sect. 1). The number of spheres was chosen in order to obtain statistically significant information while focusing on the precision of the reconstruction. We prepared three packings with different densities (see Sect. 1).

The densities were chosen in order to be representative of the upper half of the range of disordered sphere packings, between the limits defined by the Random Loose Packing (RLP) [26,27] and the Random Close Packing (RCP) $[10,27,28]$. We determined the error on the 3D localization of the reconstructed centers to be approximately $3 \cdot 10^{-3} \mathrm{D}$, commensurate with the dispersion in the spheres radius $1.6 \cdot 10^{-3} \mathrm{D}$ determined through the Archimede's method. This relative precision is comparable to other reported measurements with different techniques [15]. To avoid boundary effects, the reconstructed packings were cropped. For each sample we defined a cylindrical region of interest (ROI), the surface of which was chosen to be 4 diameters away from the packing boundaries (container walls and packing top surface) and we discarded all spheres outside the ROI (in gray in Fig. 1a). All spheres lying inside each ROI defined our entire samples respectively (in color in Fig. 1a). The samples were treated as a whole and we also analyzed $s u b$ ROIs determined by evenly dividing the entire ROI with two horizontal planes to obtain three subsamples composed of the upper, middle and lower part of the entire sample.

The mathematical and geometrical analyses of the spheres position were based on the sphere-sphere correlation func- tion $f(r)$ in Eq. 1 and on Voronoi polyhedra space decomposition respectively. Apart from a difference in average density between the subsamples, no other specific features or inhomogeneities were detected in the individual parts and we concluded that the partial volumes can be seen as individual entities with similar properties.

The radial correlation function $f(r)$ is defined as the average number of sphere centers per unit of volume at distance $r$ from a given sphere placed in the origin, rescaled by the average volume of one sphere, $V_{b}$,

$f(r)=\left\langle\frac{N_{\delta}(r) \cdot V_{b}}{V_{\delta}(r)}\right\rangle$

where $V_{\delta}(r)$ is the volume of the spherical shell of radius $r$. The shell thickness $\delta$ was adjusted to obtain a good compromise between noise and resolution (in our case $\delta \approx$ $\left.10^{-3} D\right) . N_{\delta}(r)$ was the number of sphere centers located within the distance interval $(r-\delta / 2, r+\delta / 2)$. The average value (indicated with $\langle\cdot\rangle$ ) is determined by taking into account all spheres located within the actual region of interest (ROI or $s u b \mathrm{ROI})$. The function $f(r)$ contains information about local structure, medium range order and long range average sample density. It can only be calculated for particles that are at least at a distance $r$ from the ROI borders thus significantly decreasing the quantity of useful spheres available for long range determinations, especially in small-sized ROI. Following the idea of Scott [29], we introduced a corrected correlation function $f_{c}(r)$ that overrides the limitation through the substitution of $V_{\delta}(r)$ by $V_{\delta}^{i n t}(r)$, its fraction intersecting the ROI, such as to have

$f_{c}(x)=\left\langle\frac{N_{\delta}(x) \cdot V_{b}}{V_{\delta}^{i n t}(x)}\right\rangle$

where we define $x=r / D$ to normalize the distances by the sphere diameter and thus have a general expression that is independent of the size of the spheres. This definition allows

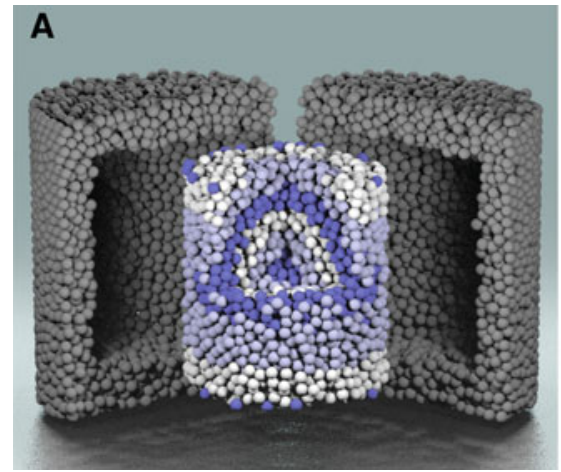

Fig. 1 a Computer-generated image of a reconstructed packing. The entire region of interest (ROI) is shown in color, the different colors corresponding to spheres at increasing distance from the ROI center. The gray spheres were discarded for the analyses. b Corrected correlation function $f_{c}(x)$ calculated for a typical sample. The features from

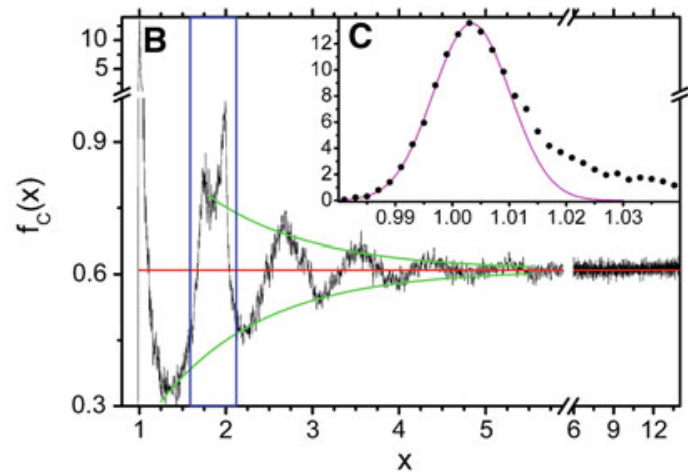

which we extract the four main parameters are highlighted in color: (1) red line average density $\rho$; (2) green line amplitude decay envelope; (3) pink line Gaussian fit of the peak at $x \approx 1$ (c); (4) blue line shape of $f_{c}(x)$ around $x \approx 2$ (color figure online) 


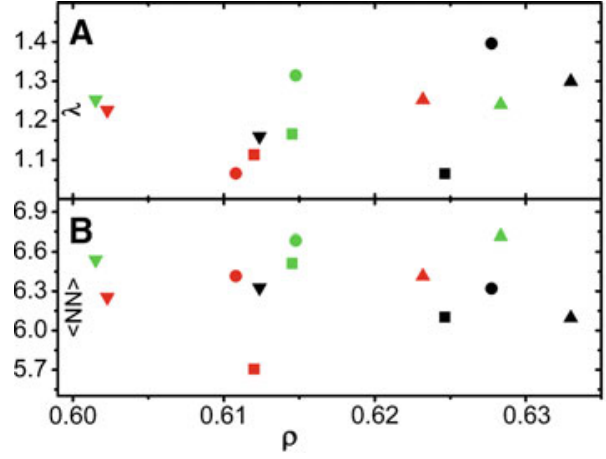

Fig. 2 a Correlation length $\lambda$, in units of spheres diameter, as a function of the average density $\rho$. b Average number of nearest neighbors $\langle N N\rangle$ as a function of the average density $\rho$. c Inverse estimated first shell volume $P_{1}^{-3}$ as a function of the average density $\rho$ (a linear fit of the data is shown in magenta). d Detail of the shape of $f_{c}^{\prime}\left(x^{\prime}\right)$ around

for a dramatical increase in statistics for large $x$ values and a decrease in distortions in the mesoscopic range.

Following the MRI acquisitions and the subsequent reconstruction and cropping procedures, we computed $f_{c}(x)$. We focused on four parameters extracted from the analysis of $f_{c}(x)$ :

- the average density $\rho$ deduced from the long-range asymptotic value of $f_{c}(x)$;

- the center of the short-range peak $(x \approx 1)$ of $f_{c}(x)$;

- the shape of $f_{c}(x)$ in the medium range $x \approx 2$;

- the envelope of $f_{c}(x)$ and in particular its decaying shape.

The average density $\rho$ was deduced by calculating the mean value of $f_{c}(x)$ after the oscillations amplitude decayed below the noise level (typically for $x>8$, see Fig. 1b-red).

Estimating the distribution of the number of mechanical neighbors of single particles (i.e. the neighbors exerting a force on them), or even its average value, from experimental data is a nontrivial problem due to the unavoidable noise present in the measurements. The method described herein cannot be used to discriminate between spheres in mechanical contact and spheres separated by a slightly larger distance and not exerting force on one another (quasi-mechanical). We define the nearest neighbors $(N N)$ as both mechanical and quasi-mechanical neighbors of a single sphere. All $N N$ contribute to the peak of $f_{c}(x)$ centered at $x \approx 1$. We observed that this peak does not appear exactly at the same value of $x$ in all samples. Let $P_{1}$ be the exact $x$ position of the center of this peak for a given sample. The value of $P_{1}$ is estimated via a Gaussian fit of the upper half of the peak to minimize the effect of the distortions appearing on the right side (Fig. 1c). Although the characterization of this peak does not allow a straightforward determination of the sphere-sphere mechanical interaction properties, it gives information on the short range geometrical structure. The value of $P_{1}^{3}$ can be used to

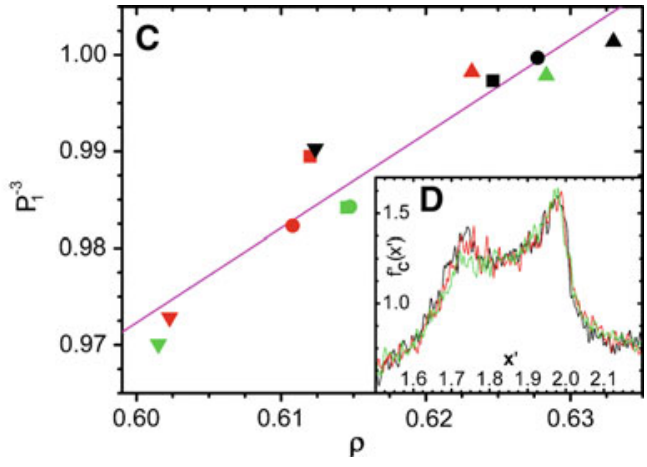

$x^{\prime} \approx 2$. Each color represents a sample (black line 1; red line 2; green line 3; see Sect. 1) and each symbols corresponds to a specific part of the samples (square entire samples, triangle upper part, circle middle part, inverted triangle lower part) (color figure online)

estimate the average volume of the first shell. The inverse of this volume, linked to the short-range correlations, was plotted for each samples and their fractional parts as a function of the average density $\rho$, which is an indicator of how beads arrange in the long-range ${ }^{1}$ (Fig. 2c). The results revealed a strong and approximately linear correlation between $P_{1}^{-3}$ and $\rho$ in the range $0.6<\rho<0.64$. The average number of nearest neighbors $\langle N N\rangle$, estimated by integrating the Gaussian fit of the peaks centered at $x=P_{1}$, shows no correlation with respect to $\rho$ (Fig. 2b). We used the two parameters $P_{1}$ and $\rho$ to rescale the correlation function and its argument in the following manner:

$f_{c}^{\prime}\left(x^{\prime}\right)=\frac{f_{c}\left(x / P_{1}\right)}{\rho}$

As shown in Fig. 2d, the rescaled correlation functions $f_{c}^{\prime}\left(x^{\prime}\right)$ match both the period and amplitude of the oscillations ${ }^{2}$ in the range $1.7<x<2.2$. Furthermore, with the proposed rescaling the overlapping of different correlation functions is improved also at larger $x$ values. The tight correlation between $P_{1}$ and the average density along with the independence of $\langle N N\rangle$ on the value of $\rho$ suggest that the difference in density between samples are not linked to changes in structural properties but rather to the average distance of the nearest neighbors which defines the size of the first shell. In this picture the volume of the first shell directly determines the long range density.

The nearly identical shape of the rescaled correlation functions at the second maximum between $1.7<x<2.2$, as well

\footnotetext{
$\overline{1} P_{1}^{-3}$ is the natural choice to perform a comparison with $\rho$, since it has itself the dimensions of a density.

${ }^{2}$ Some minor differences are still apparent, mainly in the small peaks superimposed to the oscillations at $x=\sqrt{3}$ and $x=2$. These differences can be related to samples inhomogeneities and tend to disappear as the ROI size decreases, although the noise level increases and blurs observations.
} 


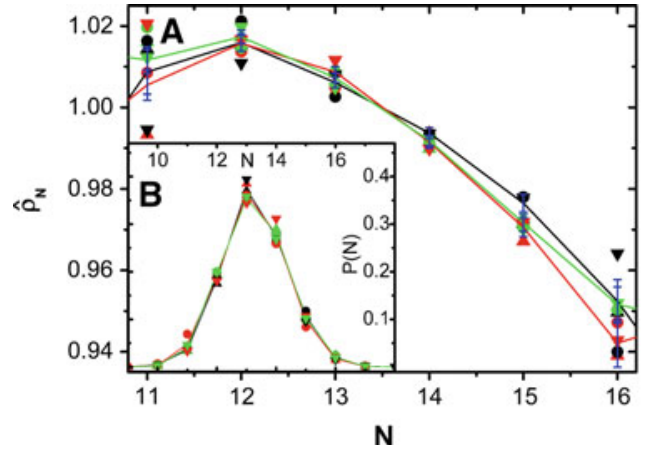

Fig. 3 a Renormalized single-particle density $\hat{\rho}_{N}$ as a function of the number of faces $N$ of the Voronoi polyhedra for all entire samples and their partial volumes (vertical bars represent standard deviation for entire samples). b Distribution $P(N)$ of the number of faces of the Voronoi polyhedra. Symbol and color codes are defined as in Fig. 2. The data corresponding to the 3 entire samples are linked by straight

as the improvement in the superposition of different correlation functions at larger $x$ values, support this hypothesis.

The damped oscillations observed in $f_{c}(x)$ for $x>1$ (see Fig. 1b-green) express the amplitude of the correlation between the sphere under investigation and the spheres located at distance $x D$. The period of the oscillations was found to be essentially constant $(\Delta x \approx 0.9)$ over the entire accessible range, only slightly increasing with increasing $x$. We determined the oscillation damping by measuring the absolute difference between the value of the oscillation amplitude extrema, $P H(x)$, and the average density $\rho$ of the samples:

$q(x)=|P H(x)-\rho|$.

The different theories proposed to describe the long-range behavior of the correlation function support either an exponential amplitude decay or a power law tail $[5,6,23,30]$. Our results showed that the main contribution to the envelope of the correlation function can be well described by an exponentially decaying function $\propto e^{-x / \lambda}$, in which $\lambda$ represents the correlation length. ${ }^{3}$ For the accessible range of our measurements, $1<x<7$, the correlation length was found to be $1.1 D<\lambda<1.35 D$ (Fig. 2a). This value did neither depend on the sample size nor on the average density.

Our analysis led to the conclusion that structural modifications of sphere packings, originate from local rearrangements at the scale of the first shell only. With the exception of the random nature of the single sphere shell geometry, the rearrangements do not change, on average, the structural

\footnotetext{
3 The exponent $p$ found with a power law fitting was in the range $1.9<p<2.5$. If extrapolated, this power law would give an infinite correlation length that is not coherent with common understanding of granular systems. Indeed we underline that this does not preclude the possibility of a different scaling law at longer ranges, nor a more complex behavior at mesoscopic ranges (e.g. a superposition of shorter scale exponential decay with a larger scale power law, etc...).
}
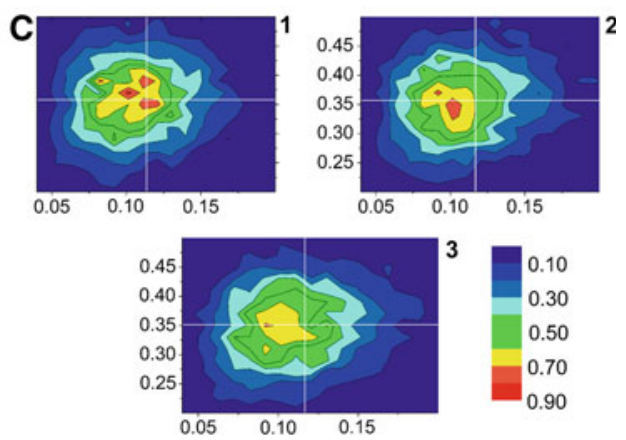

segments as guide for the eyes. c Probability density maps of the two Steinhardt structural order parameters $Q_{4}$ and $Q_{6}$ for the three entire samples; the white lines represent the average values of $Q_{4}$ and $Q_{6}$ respectively. $Q_{4}$ and $Q_{6}$ average values and standard deviations are identical for the three samples, within the error margin (color figure online)

characteristics of the sample and only lead to modifications of the average distance of the first shell nearest neighbors. These variations in distance and their effects on the volume of the first shell propagate at a mesoscopic and long range, leading to an expansion or a shrinkage of the entire sample.

To support our conclusions, a geometrical analysis based on Voronoi polyhedra [31] was performed to extract structural information from our data. Following space decomposition, we calculated the volume of each single polyhedron, $V_{i}$, and defined single-particle densities, $\rho_{i}=\frac{\pi D^{3}}{6 V_{i}}$.

The values of the Steinhardt structural order parameters $Q_{4, i}$ and $Q_{6, i}$ [32], very sensitive indicators of the presence of geometrical order, were also computed for each polyhedron. The Steinhardt parameters were computed using the presence of a common face in the Voronoi decomposition as criterion to define neighborhood. Note that in this approach, all information related to the size of the structures is contained in $\rho=\left\langle\rho_{i}\right\rangle,{ }^{4}$ whereas the details of the geometry are encoded in $Q_{4}$, in $Q_{6}$, in the distribution $P(N)$ of the number of faces of the Voronoi polyhedra, as well as in the ratios between average polyhedra volumes as a function of number of faces. Voronoi polyhedra represent the geometrical structure not only at a local scale $(x \approx 1)$ but also up to a distance of $x \approx 1.8-2.0$, mixing information related to the first shell and the mesoscopic region.

To examine the relationship between the geometry of the Voronoi polyhedra and the sample density, we defined $\rho_{N}$ as the average single-particle density for each value of $N$ and we normalized it by the global average density to obtain $\hat{\rho}_{N}=\rho_{N} / \rho$ (Fig. 3a). This is equivalent to the same rescaling which was applied to the correlation function. We observed that $\hat{\rho}_{N}$ only varies by less than $10 \%$ over the whole range

\footnotetext{
${ }^{4}$ Note that despite the different definition the value obtained from the set of $\rho_{i}$ gives the same result as the value determined from the correlation function (Fig. 1b-red), within the error margin.
} 
of $N$ in which we have a significant number of measured polyhedra $(11 \leq N \leq 16)$. The maximum of the average density is found at $N=12$. As for the rescaled correlation function $f_{c}^{\prime}\left(x^{\prime}\right)$, the renormalized single-particle density $\hat{\rho}_{N}$ is essentially identical for all samples and their subsamples. This observation leads to two important considerations; on one hand it indicates that the scaling relation is valid not just on average for $f_{c}^{\prime}\left(x^{\prime}\right)$, but also for the local structures; on the other hand, it means that the overall arrangement of the first shell, up to $\mathrm{x}=1.8$ and not just the peak at $\mathrm{x}=1$, are scaling with the density. In addition, $P(N)$ is independent on the sample density ${ }^{5}$ regardless of the sample density. Finally, the probability density maps of $\left(Q_{4, i}, Q_{6,1}\right)$ (Fig. 3c) demonstrate that no specific structure was detectable in any of the samples. The probability density was significantly larger than zero over a wide area centered around $\left(Q_{4}, Q_{6}\right)=(0.11,0.34)$. However, no accumulation of points was observed in the proximity of $\left(Q_{4}, Q_{6}\right)$ pairs that are characteristic of either hcp, fcc, bcc and sc crystals, or icosaedral structures (see e.g. [33]).

The present study reveals a strong correlation between the volume of the shell defined by the nearest neighbors (first shell) and the average density of the sample. To the best of our knowledge, this scaling relation is reported for the first time. Even if it is a well known fact for molecular liquids, it had been conjectured for granulars in the sixties [10], and subsequently abandoned. This is the first time it is directly observed in a real granular material. We believe that the high degree of monodispersion and reconstruction precision of our measurements is crucial in obtaining the present result. ${ }^{6}$ The inverse of the estimated volume of the first shell indeed showed a linear dependence on the sample density. The sphere-sphere correlation function is essentially identical for all samples if it is simply rescaled by the sample density and the average distance between nearest neighbors. This demonstrates that the compaction of sphere packings is linked to the variation of the first shell size rather than to structural rearrangements. Our measurements showed that the main contribution to the correlation amplitude decay for the mesoscopic range (up to $r / D=7$ ) can be described as an exponential rather than a power law tail. We observed

\footnotetext{
5 The slight differences that can be observed between distributions are due to noise. Indeed, if we consider a simple picture in which a single polyhedron with $N$ faces is randomly chosen from the distribution $P(N)$, the standard deviation is $\sigma(P(N))=$ $\sqrt{P(N)(1-P(N)) /(n-1)}$ with $n$ the total number of spheres in the sample. Comparing $P(N)$ from different samples we found that their difference is at most $2 \sigma$.

${ }^{6}$ Since the correlation we found between $\rho$ and $P_{1}$, resides on a very narrow region $\left(P_{1}^{-3}\right.$ varies by less than $0.04 D^{-3}$ meaning $P_{1}$ changes of about $0.013 D$ ) we believe that this effect could not be detectable without a so high bead monodispersion as we have (to make a comparison the bead diameter dispersion in [15] $1.59 \pm 0.05 \mathrm{~mm}$ corresponds to about 3 times the full vertical axis of Fig. 2c).
}

no sign of accumulation in the probability density maps of the two Steinhardt structural order parameters $Q_{4}$ and $Q_{6}$ around typical values of regular lattices. We thus conclude that no regular crystalline order was present in the samples. Moreover, no accumulation of $\left(Q_{4}, Q_{6}\right)$ pairs was detected, suggesting the absence of statistically significant quasicrystalline or non-crystalline structures with defined values of $Q_{4}$ and $Q_{6}$. In dry packed samples below the 0.64 density limit, Aste et al. [15] observed the presence of $\left(Q_{4}, Q_{6}\right)$ pairs close to the values expected for perfect fcc and hcp crystals. They estimated the occurrence of these pairs to be even greater than $10 \%$.

Although our criterion to define neighbors for $Q_{4}$ and $Q_{6}$ calculation differs from the one used in [15], in our conditions of high monodispersion and low friction due to the aqueous environment we did not find any $\left(Q_{4}, Q_{6}\right)$ pair close to crystalline-like values. The lack of cristallinity in our samples is in agreement with previous observations on packings below the RCP density. If the packing density is below the RCP limit, crystallization is not expected both in the case of simulated frictionless ideal spheres $[34,35]$ and in the case of frictional experimental data on dry packings [36]. We should also underline that in contrast with our system, colloidal system do show crystallization in most cases [37]. In the latter case, however, capillary forces modify considerably the force chains. We stress that the high degree of monodispersion and reconstruction precision of our measurements are crucial in obtaining our result.

Our methods and findings opened new perspectives for investigating the nature and properties of disordered sphere packing, both from an experimental and an interpretative point of view. Novel approaches for optimization problems, information theory and the study of the jamming transition could be designed and we can foresee countless potential industrial applications in which powders or grains require compaction or handling.

\section{Materials and methods}

The packings considered in this work were composed of sharply monodisperse, diameter $D=3.015 \mathrm{~mm}$ plastic spheres (Polyoxymethylene, R.P.G. International, Italy). The particle volume distribution has been measured through the Archimede's method, giving a standard error on the diameter of $0.005 \mathrm{~mm}$. The spheres were placed in a vertical plastic cylinder, filled with a contrast medium (water containing a concentration of $40 \mathrm{mM} \mathrm{CuSO}_{4}$ ). All packings were prepared by slowly pouring the spheres (approximately 20,000) in the container prefilled with the solution, carefully avoiding the presence of air bubbles. Prior to perform the MRI measurements, the packings were vertically vibrated using sinusoidal agitation at different frequencies in the range $40-1,000 \mathrm{~Hz}$, 
with average acceleration modulus $1 g<\sqrt{\left\langle\ddot{z}^{2}(t)\right\rangle}<3 g$, and avoiding convection (aging procedure). Packing 1 was aged for $48 \mathrm{~h}$, packing 2 for $2 \mathrm{~h}$, and packing 3 for $30 \mathrm{~min}$ (numbers corresponds to those displayed in Figs. 2 and 3). All other parameters were kept identical.

The packings were scanned in a Siemens 7T clinical scanner using a birdcage MRI volume coil (designed to accommodate a human head). The 3D images were then acquired using a standard Gradient Echo Imaging (GRE) technique ( $T R=50 \mathrm{~ms}, T E=3.79 \mathrm{~ms}$, total acquisition time of $6 \mathrm{~h}$ ). The output 3D matrix consisted of a water density map with an isotropic resolution of $0.2 \mathrm{~mm}$. After having applied a threshold on the amplitude of each voxel, the tomographies were Hough transformed to produce spatially separated sets of voxels associated to each sphere. The position of each sphere was then determined from the mass center of the corresponding set of voxels. Several postprocessing checks were performed to ensure the complete reconstruction of the packings (every single sphere was mapped). We estimated the error on the 3D localization of the reconstructed centers to be on the order of $3 \cdot 10^{-3} \mathrm{D}$, commensurate with the dispersion in the spheres radius $1.6 \cdot 10^{-3} \mathrm{D}$. From a simple error propagation calculation, the expected standard error on the Gaussian peak at $x=1$ should be about $4.8 \cdot 10^{-3} D$. This value is in very good agreement with all the observed correlation functions. Due to the extreme monodispersion in the sphere diameters, the standard deviation is dominated by the reconstruction precision.

In order to avoid boundary effects, we discarded the spheres placed at a distance lower than four diameters from the container walls (gray spheres in Fig. 1a). Our entire region of interest (ROI) typically contained about 4,500 spheres in a cylindrical volume of diameter $11 D$ and height $15 D$ (colored spheres in Fig. 1a). The subsamples were defined by dividing the entire ROIs with horizontal planes in three non overlapping parts of identical volume.

Acknowledgments R. B. and A. L. S. would like to thank A. Magill for technical help.

Competing interests The authors declare that they have no competing financial interests. This work was supported by the Swiss National Science Foundation (grant no. PPOOP2 133562 to A. C. and 200020_126534 to G. G.), the Centre d'Imagerie BioMédicale (CIBM) of the UNIL, UNIGE, HUG, CHUV, EPFL, and the Leenards and Jeantet Foundations.

\section{References}

1. Brown, R.L., Richards, J.C.: Principles of Powder Mechanics, Volume 10 of International Series of Monographs in Chemical Engineering. Pergamon Press, NY (1970)

2. Nedderman, R.M.: Statics and Kinematics of Granular Materials. Cambridge University Press, Cambridge (1992)
3. Jaeger, H.M., Nagel, S.R., Behringer, R.P.: Granular solids, liquids, and gases. Rev. Mod. Phys. 68(4), 1259-1273 (1996)

4. Jensen, H.J.: Self-Organized Criticality: Emergent Complex Behavior in Physical and Biological Systems. Cambridge Lecture Notes in Physics. Cambridge University Press, Cambridge (1998)

5. Dauchot, O.: Glassy behaviours in a-thermal systems, the case of granular media: a tentative review. In: Henkel, M., Pleimling, M., Sanctuary, R. (eds.) Ageing and the Glass Transition, vol. 716 of Lecture Notes in Physics, pp. 161-206. Springer, Berlin (2007)

6. Kawaguchi, T.: Mri measurement of granular flows and fluidparticle flows. Adv. Powder Technol 21(3), 235-241 (2010)

7. Sellerio, A.L., Mari, D., Gremaud, G., D'Anna, G.: Glass transition associated with the jamming of vibrated granular matter. Phys. Rev. E: Stat. Nonlinear Soft Matter Phys. 83(2), 021301 (2011)

8. Stillinger, F.H., Weber, T.A.: Packing structures and transitions in liquids and solids. Science 225(4666), 983-989 (1984)

9. Hales, T.C.: The kepler conjecture. Retrieved online, July 2011 (Nov 1998)

10. Bernal, J.D., Mason, J.: Packing of spheres: co-ordination of randomly packed spheres. Nature 188(4754), 910-911 (1960)

11. Scott, G.D.: Packing of equal spheres. Nature 188(4754), 908-909 (1960)

12. Behringer, R.P., Jenkins, J.T.: Powders and Grains 97. Balkema (1997)

13. Duran, J.: Sands, Powders, and Grains: An Introduction to the Physics of Granular Materials. Partially Ordered Systems. Springer, New York (2000)

14. Ehrichs, E.E., Jaeger, H.M., Karczmar, G.S., Knight, J.B., Kuperman, V.Y., Nagel, S.R.: Granular convection observed by magnetic resonance imaging. Science 267(5204), 1632-1634 (1995)

15. Aste, T., Saadatfar, M., Sakellariou, A., Senden, T.J.: Investigating the geometrical structure of disordered sphere packings. Phys. A: Stat. Mech. Appl. 339(1-2), 16-23 (2004) [Proceedings of the International Conference New Materials and Complexity]

16. Jerkins, M., Schröter, M., Swinney, H.L., Senden, T.J., Saadatfar, M., Aste, T.: Onset of mechanical stability in random packings of frictional spheres. Phys. Rev. Lett. 101, 018301 (2008)

17. Delaney, G.W., Di Matteo, T., Aste, T.: Combining tomographic imaging and DEM simulations to investigate the structure of experimental sphere packings. Soft Matter 6, 2992-3006 (2010)

18. Song, C., Wang, P., Makse, H.A.: A phase diagram for jammed matter. Nature 453(7195), 629-632 (2008)

19. Edwards, S.F., Grinev, D.V., Brujic, J.: Fundamental problems in statistical physics of jammed packings. Phys. A: Stat. Mech. Appl. 330(1-2), 61-76, (2003). [RANDOMNESS AND COMPLEXITY: Proceedings of the International Workshop in honor of Shlomo Havlin's 60th birthday]

20. Parisi, G., Zamponi, F.: Mean-field theory of hard sphere glasses and jamming. Rev. Mod Phys. 82, 789-845 (2010)

21. Edwards, S.F., Oakeshott, R.B.S.: Theory of powders. Phys. A: Stat. Theor. Phys. 157(3), 1080-1090 (1989)

22. Debenedetti, P.G., Stillinger, F.H.: Supercooled liquids and the glass transition. Nature 410(6825), 259-267 (2001)

23. Donev, A., Stillinger, F.H., Torquato, S.: Unexpected density fluctuations in jammed disordered sphere packings. Phys. Rev. Lett. 95, 090604 (2005)

24. Donev, A., Torquato, S., Stillinger, F.H.: Pair correlation function characteristics of nearly jammed disordered and ordered hardsphere packings. Phys. Rev. E 71, 011105 (2005)

25. Liang, Z.P., Lauterbur, P.C.: IEEE Engineering in Medicine and Biology Society. Principles of Magnetic Resonance Imaging: a Signal Processing Perspective. IEEE Press Series in Biomedical Engineering. SPIE Optical Engineering Press (2000) 
26. Onoda, G.Y., Liniger, E.G.: Random loose packings of uniform spheres and the dilatancy onset. Phys. Rev. Lett. 64(22), 27272730 (1990)

27. Berryman, J.G.: Random close packing of hard spheres and disks. Phys. Rev. A 27, 1053-1061 (1983)

28. Scott, G.D., Kilgour, D.M.: The density of random close packing of spheres. J. Phys. D: Appl. Phys. 2(6), 863 (1969)

29. Scott, G.D.: Radial distribution functions from small packings of spheres. Nature 217, 733-735 (1968)

30. Hopkins, A.B., Stillinger, F.H., Torquato, S.: Nonequilibrium static diverging length scales on approaching a prototypical model glassy state. Phys. Rev. E 86, 021505 (2012)

31. Voronoi, G.: Nouvelles applications des paramètres continus à la théorie des formes quadratiques. deuxième mémoire. recherches sur les parallélloèdres primitifs. J. für die reine und angewandte Mathematik 134, 198-278 (1908)

32. Steinhardt, P.J., Nelson, D.R., Ronchetti, M.: Bond-orientational order in liquids and glasses. Phys. Rev. B 28, 784-805 (1983)
33. ten Wolde, P.R., Ruiz-Montero, M.J., Frenkel, D.: Numerical calculation of the rate of crystal nucleation in a lennard-jones system at moderate undercooling. J. Chem. Phys. 104(24), 9932-9947 (1996)

34. Kapfer, S.C., Mickel, W., Mecke, K., Schröder-Turk, G.E.: Jammed spheres: minkowski tensors reveal onset of local crystallinity. Phys. Rev. E 85, 030301(R) (2012)

35. Bargieł, M., Tory, E.M.: Packing fraction and measures of disorder of ultradense irregular packings of equal spheres. II. Transition from dense random packing. Adv. Powder Technol., 12(4), 533557 (2001). doi:10.1163/15685520152756660

36. Schröder-Turk, G.E., Mickel, W., Schröter, M., Delaney, G.W., Saadatfar, M., Senden, T.J., Mecke, K., Aste, T.: Disordered spherical bead packs are anisotropic. EPL 90, 34001 (2010)

37. Kurita, R., Weeks, E.R.: Experimental study of random-closepacked colloidal particles. Phys. Rev. E 82, 011403 (2010) 